\title{
Mapping Peering Interconnections to a Facility
}

\author{
Vasileios Giotsas \\ CAIDA / UC San Diego \\ vgiotsas@caida.org
}

\author{
Georgios Smaragdakis \\ MIT / TU Berlin \\ gsmaragd@csail.mit.edu
}

\author{
Bradley Huffaker \\ CAIDA / UC San Diego \\ bhuffake@caida.org
}

\author{
Matthew Luckie \\ University of Waikato \\ mjl@wand.net.nz
}

\author{
kc claffy \\ CAIDA / UC San Diego \\ kc@caida.org
}

\begin{abstract}
Annotating Internet interconnections with robust physical coordinates at the level of a building facilitates network management including interdomain troubleshooting, but also has practical value for helping to locate points of attacks, congestion, or instability on the Internet. But, like most other aspects of Internet interconnection, its geophysical locus is generally not public; the facility used for a given link must be inferred to construct a macroscopic map of peering. We develop a methodology, called constrained facility search, to infer the physical interconnection facility where an interconnection occurs among all possible candidates. We rely on publicly available data about the presence of networks at different facilities, and execute traceroute measurements from more than 8,500 available measurement servers scattered around the world to identify the technical approach used to establish an interconnection. A key insight of our method is that inference of the technical approach for an interconnection sufficiently constrains the number of candidate facilities such that it is often possible to identify the specific facility where a given interconnection occurs. Validation via private communication with operators confirms the accuracy of our method, which outperforms heuristics based on naming schemes and IP geolocation. Our study also reveals the multiple roles that routers play at interconnection facilities; in many cases the same router implements both private interconnections and public peerings, in some cases via multiple Internet exchange points. Our study also sheds light on peering engineering strategies used by different types of networks around the globe.
\end{abstract}

Permission to make digital or hard copies of all or part of this work for personal or classroom use is granted without fee provided that copies are not made or distributed for profit or commercial advantage and that copies bear this notice and the full citation on the first page. Copyrights for components of this work owned by others than ACM must be honored. Abstracting with credit is permitted. To copy otherwise, or republish, to post on servers or to redistribute to lists, requires prior specific permission and/or a fee. Request permissions from permissions@acm.org.

CoNEXT'15 December 01-04, 2015, Heidelberg, Germany

(c) 2015 ACM. ISBN 978-1-4503-3412-9/15/12 . \$ \$15.00

DOI: $10.1145 / 2716281.2836122$

\section{CCS Concepts}

-Networks $\rightarrow$ Network measurement; Physical topologies;

\section{Keywords}

Interconnections; peering facilities; Internet mapping

\section{INTRODUCTION}

Measuring and modeling the Internet topology at the logical layer of network interconnection, i. e., autonomous systems (AS) peering, has been an active area for nearly two decades. While AS-level mapping has been an important step to understanding the uncoordinated formation and resulting structure of the Internet, it abstracts a much richer Internet connectivity map. For example, two networks may interconnect at multiple physical locations around the globe, or even at multiple locations in the same city $[55,49]$.

There is currently no comprehensive mapping of interconnections to physical locations where they occur [61]. There are good reasons for the dearth of this information: evolving complexity and scale of networking infrastructure, information hiding properties of the routing system (BGP), security and commercial sensitivities, and lack of incentives to gather or share data. But this opacity of the Internet infrastructure hinders research and development efforts as well as network management. For example, annotating peering interconnections with robust physical coordinates at the level of a building facilitates network troubleshooting and diagnosing attacks [43] and congestion [46]. Knowledge of geophysical locations of interconnections also enables assessment of the resilience of interconnections in the event of natural disasters [53,20], facility or router outages [6], peering disputes [46], and denial of service attacks $[22,60]$. This information can also elucidate the role of emerging entities, e. g., colocation facilities, carrier hotels, and Internet exchange points (IXP), that enable today's richly interconnected ecosystem [44, 7, $18,19,15]$. It also increases traffic flow transparency, e. g., to identify unwanted transit paths through specific 
countries, and inform peering decisions in a competitive interconnection market.

In this paper we describe a measurement and inference methodology to map a given interconnection to a physical facility. We first create and update a detailed map of interconnection facilities and the networks present at them. This map requires manual assembly, but fortunately the information is increasingly publicly available in recent years, partly due to the fact that many networks require it be available in order to establish peering [4], and many IXPs publish information about which networks are present at which of their facilities in order to attract participating networks [18]. Interconnection facilities also increasingly make the list of participant members available in their website or in PeeringDB [45]. While it is a substantial investment of time to keep such a list current, we find it is feasible.

However, a well-maintained mapping of networks to facilities does not guarantee the ability to accurately map all interconnections involving two ASes to specific physical facilities, since many networks peer at multiple locations even within a city. Mapping a single interconnection to a facility is a search problem with a potentially large solution space; however, additional constraints can narrow the search. The contributions of this work are as follows:

- We introduce and apply a measurement methodology, called constrained facility search, which infers the physical facilities where two ASes interconnect from among all (sometimes dozens of) possible candidates, and also infers the interconnection method, e.g. public peering at an IXP, private peering via cross-connect, point-to-point connection tunnelled over an IXP, or remote peering.

- We validate the accuracy of our methodology using direct feedback, BGP communities, DNS records, and IXP websites and find our algorithm achieves at least $90 \%$ accuracy for each type of interconnection and outperforms heuristics based on naming schemes and IP geolocation.

- We demonstrate our methodology using case studies of a diverse set of interconnections involving content providers (Google, Akamai, Yahoo, Limelight, and Cloudflare) as well as transit providers (Level3, Cogent, Deutsche Telekom, Telia, and NTT). Our study reveals the multiple roles that routers play at interconnection facilities; frequently the same router implements both public and private peering in some cases via multiple facilities.

\section{BACKGROUND AND TERMINOLOGY}

Interconnection is a collection of business practices and technical mechanisms that allows individually managed networks (ASes) to exchange traffic [11]. The two primary forms of interconnection are transit, when one AS sells another ISP access to the global Internet, and peering, when two ISPs interconnect to exchange customer traffic, although complicated relationships exist $[28,31]$. Whether and how to interconnect requires careful consideration, and depends on traffic volume exchanged between the networks, their customer demographics, peering and security policies, and the cost to maintain the interconnection [50].

Interconnection Facility. An interconnection facility is a physical location (a building or part of one) that supports interconnection of networks. These facilities lease customers secure space to locate and operate network equipment. They also provide power, cooling, fire protection, dedicated cabling to support different types of network connection, and in many cases administrative support. Large companies such as Equinix [27], Telehouse [59], and Interxion [36] operate such facilities around the globe. Smaller companies operate interconnection facilities in a geographic region or a city. Most interconnection facilities are carrier-neutral, although some are operated by carriers, e.g., Level3. In large communication hubs, such as in large cities, an interconnection facility operator may operate multiple facilities in the same city, and connect them, so that networks participating at one facility can access networks at another facility in the same city.

Internet Exchange Point. An Internet Exchange Point (IXP) is a physical infrastructure composed of layer-2 Ethernet switches where participating networks can interconnect their routers using the switch fabric. At every IXP there is one or more (for redundancy) high-end switches called core switches (the center switch in Figure 1). IXPs partner with interconnection facilities in the city they operate and install access switches there (switches at facilities 1 and 2 in Figure 1). These switches connect via high bandwidth connections to the core switches. In order to scale, some IXPs connect multiple access switches to back-haul switches. The backhaul switch then connects to the core switch. All IXP members connected to the same access switch or backhaul switch exchange traffic locally if they peer; the rest exchange traffic via the core switch. Thus, routers owned by members of IXPs may be located at different facilities associated with the same IXP [18].

Popular peering engineering options today are:

Private Peering with Cross-connect. A crossconnect is a piece of circuit-switched network equipment that physically connects the interfaces of two networks at the interconnection facility. It can be either copper or fiber with data speeds up to tens of Gbps. Crossconnects can be established between members that host their network equipment in different facilities of the same interconnection facility operator, if these facilities are interconnected. The downside of a cross-connect is operational overhead: it is largely a manual process to establish, update, or replace one.

Some large facilities have thousands of cross-connects, e. g., Equinix reported $161.7 \mathrm{~K}$ cross-connects across all its colocation facilities, with more than half in the Amer- 


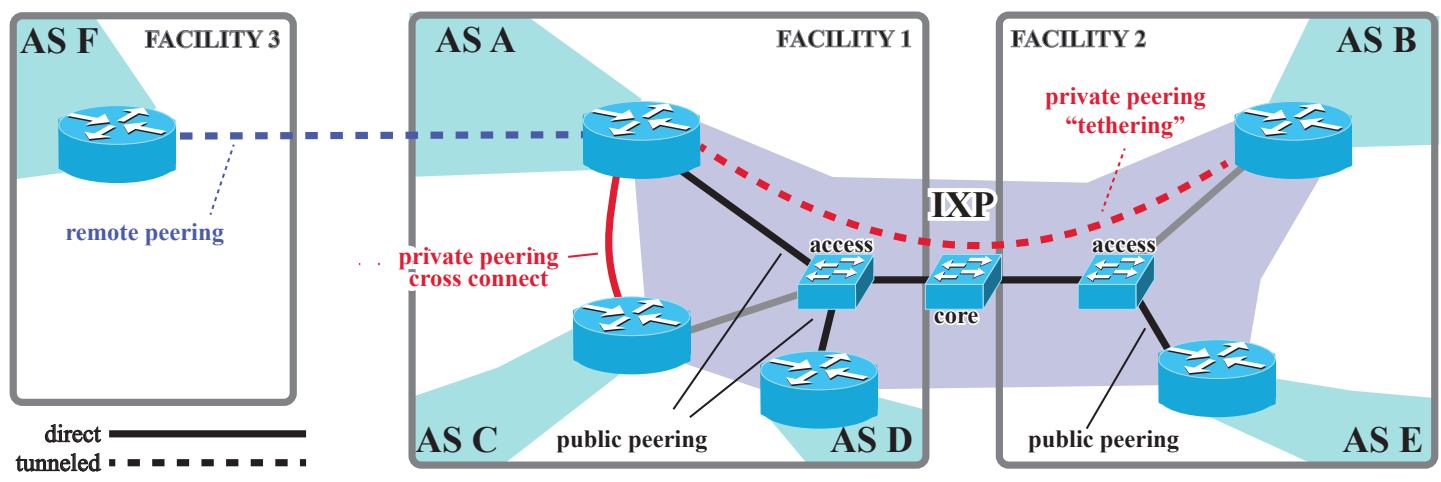

Figure 1: Interconnection facilities host routers of many different networks and partner with IXPs to support different types of interconnection, including cross-connects (private peering with dedicated medium), public peering (peering established over shared switching fabric), tethering (private peering using VLAN on shared switching fabric), and remote peering (transport to IXP provided by reseller).

icas (Q2 2015) [3]. Cross-connects are installed by the interconnection facilities but members of IXPs can order cross-connects via the IXP for partnered interconnection facilities, in some cases with a discount. For example DE-CIX in Frankfurt has facilitated more than 900 cross-connects as of February 2015 [2].

Public Peering. Public peering, also referred to as public interconnect, is the establishment of peering connections between two members of an IXP via the IXP's switch fabric. IXPs are allocated IP prefix(es) and often an AS number by a Regional Internet Registry. The IXP assigns an IP from this range to the IXP-facing router interfaces of its IXP members to enable peering over its switch fabric [10]. One way to establish connectivity between two ASes is to establish a direct BGP session between two of their respective border routers. Thus, if two IXP member ASes wanted to exchange traffic via the IXP's switching fabric, they establish a bi-lateral BGP peering session at the IXP. An increasing number of IXPs offer their members the use of route server to establish multi-lateral peering to simplify public peering $[32,54]$. With multi-lateral peering an IXP member establishes a single BGP session to the IXP's route server and receives routes from other participants using the route server. The advantage of public peering is that by leasing one IXP port it is possible to exchange traffic with potentially a large fraction of the IXP members [57].

Private Interconnects over IXP. An increasing number of IXPs offer private interconnects over their public switch fabric. This type of private peering is also called tethering or IXP metro VLAN. With tethering, a point-to-point virtual private line is established via the already leased port to reach other members of the IXP via a virtual local area network (VLAN), e.g., IEEE 802.1Q. Typically there is a setup cost. In some cases this type of private interconnect enables members of an IXP to privately reach networks located in other facilities where those members are not present, e.g., transit providers or customers, or to privately connect their infrastructure across many facilities.

Remote Peering. Primarily larger IXPs, but also some smaller ones, have agreements with partners, e.g., transport networks, to allow remote peering [14]. In this case, the router of the remote peer can be located anywhere in the world and connects to the IXP via an Ethernet-over-MPLS connection. An advantage of remote peering is that it does not require maintaining network equipment at the remote interconnection facilities. Approximately 20\% (and growing) of AMS-IX participants were connected this way [18] in 2013. Remote peering is also possible between a remote router at the PoP of an ISP and a router present at an interconnection facility.

\section{DATASETS AND MEASUREMENTS}

To infer details of a given interconnection, we need information about the prefixes of the two networks and physical facilities where they are present. This section describes the publicly available data that we collected and analyzed for this study, and the publicly available measurement servers (vantage points) we utilized.

\subsection{Data Sources}

\subsubsection{Facility Information}

For a given network we developed (and continue to maintain to keep current) a list of the interconnection facilities where it is present. Despite the fact that facilities for commercial usage must be known to the network operators to facilitate the establishment of new peering links and to attract new customers, and in some cases it is required to be public (e.g., for facilities that partner with IXPs in Europe), the information is not available in one form.

We started by compiling an AS-to-facilities mapping using the list of interconnection facilities and associated networks (ASNs) available in PeeringDB [45]. Al- 


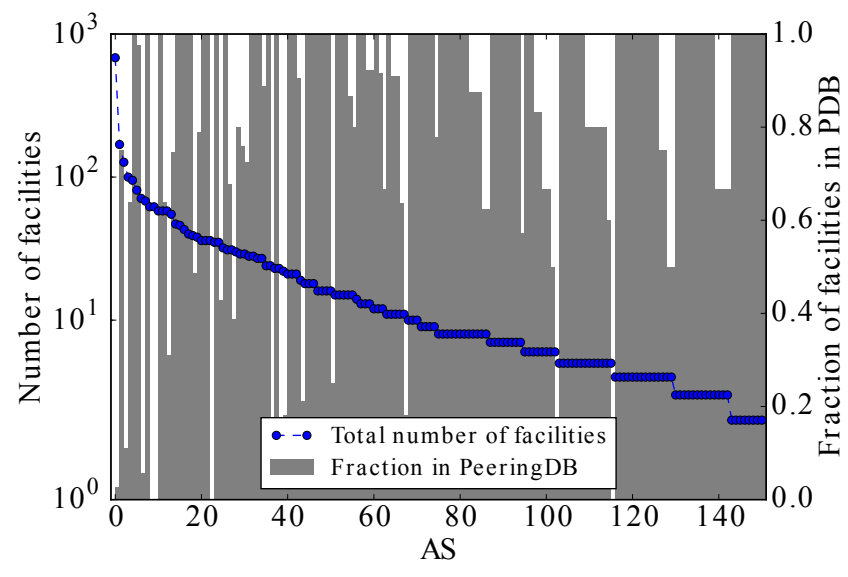

Figure 2: Number of interconnection facilities for 152 ASes extracted from their official website, and the associated fraction of facilities that appear in PeeringDB.

though this list is maintained on a volunteer basis (operators contribute information for their own networks), and may not be regularly updated for some networks, it is the most widely used source of peering information among operators, and it allows us to bootstrap our algorithms. Due to its manual compilation process, there are cases where different naming schemes are used for the same city or country. To remove such discrepancies, we convert country and city names to standard ISO and UN names. If the distance between two cities is less than 5 miles, we map them to the same metropolitan area. We calculate the distance by translating the postcodes of the facilities to geographical coordinates. For example, we group Jersey City and New York City into the NYC metropolitan area.

To augment the list of collected facilities, we extracted colocation information from web pages of Network Operating Centers (NOCs), where AS operators often document their peering interconnection facilities. Extracting information from these individual websites is a tedious process, so we did so only for the subset of networks that we encountered in our traceroutes and for a network's PeeringDB data did not seem to reflect the geographic scope reported on the network's own web site. For example, we investigated cases where a NOC web site identified a given AS as global but PeeringDB listed facilities for that AS only in a single country.

Figure 2 summarizes the additional information obtained from NOC websites. The gray bars show the fraction of facilities found in PeeringDB. We checked 152 ASes with PeeringDB records, and found that PeeringDB misses 1,424 AS-to-facility links for 61 ASes; for 4 of these ASes PeeringDB did not list any facility. Interestingly, the ASes with missing PeeringDB information provided detailed data on their NOC websites, meaning that they were not intending to hide their presence.

\subsubsection{IXP Information}

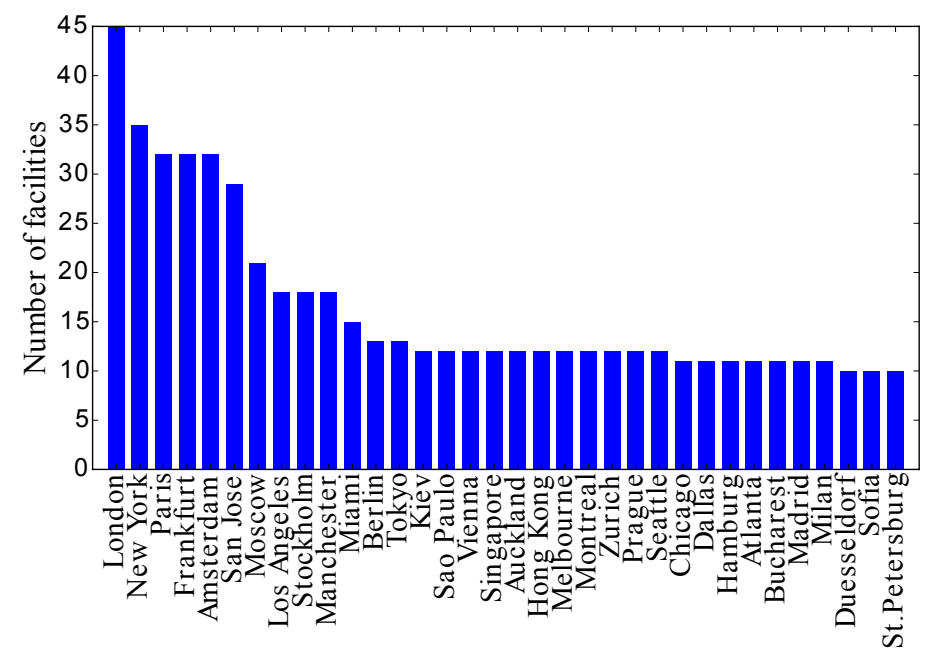

Figure 3: Metropolitan areas with at least 10 interconnection facilities.

We use various publicly available sources to get an up-to-date list of IXPs, their prefixes, and associated interconnection facilities. This information is largely available from IXP websites. We also use lists from PeeringDB and Packet Clearing House (PCH). Useful lists are provided by regional consortia of IXPs such as Euro-IX (also lists IXPs in North America), Af-IX, LAC-IX, and APIX that maintain databases for the affiliated IXPs and their members. Some IXPs may be inactive; PCH regularly updates their list and annotates inactive IXPs. To further filter out inactive IXPs, for our study, we consider only IXPs that (i) we were able to confirm the IXP IP address blocks from at least three of these data sources, and (ii) we could associate at least one active member from at least two of the above data sources. We ended up with 368 IXPs in 263 cities in 87 countries. IXPs belonging to the same operators in different cities may be different entries, e.g., DE-CIX Frankfurt and DE-CIX Munich.

We then create a list of IXPs where a network is a member, and annotate which facilities partner with these exchange points. For private facilities, we use PeeringDB data augmented with information available at the IXP websites and databases of the IXP consortia. We again encountered cases where missing IXP information from PeeringDB was found on IXP websites. For example, the PeeringDB record of the JPNAP Tokyo I exchange does not list any partner colocation facilities, while the JPNAP website lists two facilities [5]. Overall, we extracted additional data from IXP websites for 20 IXPs that we encountered in traces but for which PeeringDB did not list any interconnection facilities. PeeringDB was not missing the records of the facilities, only their association with the IXPs.

By combining all the information we collected for facilities, we compiled a list of 1,694 facilities in 95 countries and 684 cities for April 2015. The regional distribution of the facilities is as follows: 503 in North America, 860 in Europe, 143 in Asia, 84 in Oceania, 73 
in South America, and 31 in Africa. Notice that these facilities can be operated by colocation operators or by carriers. Figure 3 shows the cities with at least 10 colocation facilities. It is evident that for large metropolitan areas the problem of pinpointing a router's PoP at the granularity of interconnection facility is considerably more challenging than determining PoP locations at a city-level granularity.

On average a metropolitan area has about 3 times more interconnection facilities than IXPs. This happens because the infrastructure of IXPs is often distributed among several facilities in a city, or even across neighboring cities, for purposes of redundancy and expanded geographical coverage. For example, the topology of DE-CIX in Frankfurt spans 18 interconnection facilities. ASes tend to connect to more interconnection facilities than IXPs, with $54 \%$ of the ASes in our dataset connected to more than one IXPs and $66 \%$ of the ASes connected at more than one interconnection facilities. This may be intuitive since connectivity to an IXP requires presence at least one interconnection facility that partners with the IXP. However, we observe the opposite behavior for a relatively small number of ASes that use fewer than 10 interconnection facilities. This behavior is consistent with two aspects of the peering ecosystem: (i) an interconnection facility may partner with multiple IXPs, so presence at one facility could allow connectivity to multiple IXPs, and (ii) remote peering allows connectivity to an IXP through an IXP port reseller, in which case presence at an IXP does not necessarily require physical presence at one of its partner facilities. For instance, about $20 \%$ of all AMS-IX participants connect remotely [18].

\subsection{Vantage Points and Measurements}

To perform targeted traceroute campaigns we used publicly available traceroute servers, RIPE Atlas, and looking glasses. We augmented our study with existing daily measurements, from iPlane and CAIDA's Archipelago infrastructures, that in some cases had already traversed interconnections we considered. Table 1 summarizes characteristics of our vantage points.

RIPE Atlas. RIPE Atlas is an open distributed Internet measurement platform that relies on measurement devices connected directly to home routers, and a smaller set of powerful measurement collectors (anchors) used for heavy measurements and synchronization of the distributed measurement infrastructure. The end-host devices can be scheduled to perform traceroute, ping, and DNS resolution on the host. We employed ICMP Paris (supported by RIPE Atlas) traceroute to mitigate traceroute artifacts caused by load balancing [9]. We also used existing public measurements gathered previously by RIPE Atlas nodes (e. g., periodic traceroute queries to Google from all Atlas nodes).

Looking Glasses. A looking glass provides a webbased or telnet interface to a router and allows the execution of non-privileged debugging commands. In

\begin{tabular}{l|cccc|c}
\hline & $\begin{array}{c}\text { RIPE } \\
\text { Atlas }\end{array}$ & LGs & iPlane & Ark & $\begin{array}{c}\text { Total } \\
\text { unique }\end{array}$ \\
\hline Vantage Pts. & 6385 & 1877 & 147 & 107 & 8517 \\
ASNs & 2410 & 438 & 117 & 71 & 2638 \\
Countries & 160 & 79 & 35 & 41 & 170 \\
\hline
\end{tabular}

Table 1: Characteristics of the four traceroute measurement platforms we utilized.

many cases a looking glass provides access to routers in different cities, as well multiple sites at the same city. Many looking glasses are also colocated with IXPs. Often looking glass operators enforce probing limitations through mandatory timeouts or by blocking users who exceed the operator-supported probing rate. Therefore, looking glasses are appropriate only for targeted queries and not for scanning a large range of addresses. To conform to the probing rate limits, we used a timeout of 60 seconds between each query to the same looking glass.

We extracted publicly available and traceroute-capable looking glasses from PeeringDB, traceroute.org [39], and previous studies [41]. After filtering out inactive or otherwise unavailable looking glasses, we ended up with 1877 looking glasses in 438 ASes and 472 cities including many in members of IXPs and 21 offered by IXPs. An increasing number of networks run public looking glass servers capable of issuing BGP queries [32], e.g., "show ip bgp summary", "prefix info", "neighbor info". We identified 168 that support such queries and we used them to augment our measurements. These types of looking glasses allow us to list the BGP sessions established with the router running the looking glass, and indicate the ASN and IP address of the peering router, as well as showing metainformation about the interconnection, e. g., via BGP communities [31].

iPlane. The iPlane project [47] performs daily IPv4 traceroute campaigns from around 300 PlanetLab nodes. iPlane employs Paris traceroute to target other PlanetLab nodes and a random fraction of the advertised address space. We used two daily archives of traceroute measurements, collected a week apart, from all the active nodes at the time of our measurements.

CAIDA Archipelago (Ark). CAIDA maintains Ark, a globally distributed measurement platform with 107 nodes deployed in 92 cities (as of May 2015 when we gathered the data). These monitors are divided into three teams, each of which performs Paris traceroutes to a randomly selected IP address in every announced $/ 24$ network in the advertised address space in about 2-3 days. We analyzed one dataset collected when we performed the traceroute campaigns with RIPE Atlas and the looking glasses.

Targeted traceroutes. It takes about 5 minutes for a full traceroute campaign using more than $95 \%$ of all active RIPE Atlas nodes for one target. The time required by each looking glass to complete a traceroute measurement to a single target depends on the number of locations provided by each looking glass. The largest 
looking glass in our list has 120 locations. Since we wait 60 seconds between queries, the maximum completion time is about 180 minutes, assuming that a traceroute takes about 30 seconds to complete.

\section{METHODOLOGY}

Next we describe the technique we designed and implemented to infer the location of an interconnection. Figure 4 depicts the overall methodology and datasets used at each step of the inference process.

\subsection{Preparation of traceroute data}

Interconnections occur at the network layer when two networks agree to peer and exchange traffic. To capture these interconnections, we performed a campaign of traceroute measurements from RIPE Atlas and looking glass vantage points, targeting a set of various networks that include major content providers and Tier-1 networks (see section 5). The collected traceroute data is augmented with archived traceroute measurements performed by iPlane and CAIDA Ark, and when relevant from RIPE Atlas archived measurements (Table 1).

We first mapped each IP router interface to an ASN using Team Cymru's IP-to-ASN service [21], which utilizes multiple BGP sources to construct this mapping. Mapping IP addresses to ASNs based on longest prefix matching is prone to errors caused by IP address sharing between siblings or neighboring ASes [63]. Such errors can reduce the accuracy of our methodology since they can lead to inference of incorrect candidate facilities for an IP interface. To reduce common errors, we detect potentially erroneous IP to ASN mappings by performing alias resolution to group IP interfaces into routers. We resolved 25,756 peering interfaces and found 2,895 alias sets containing 10,952 addresses, and 240 alias sets that included 1,138 interfaces with conflicting IP to ASN mapping. We map alias sets with conflicting IP interfaces to the ASN to which the majority of interfaces are mapped, as proposed in [16].

We used the MIDAR alias resolution system [40] to infer which aliases belong to the same router. MIDAR uses the monotonic bounds test on a sequence of collected IP-ID values to infer which IP addresses are aliases, and has been shown to produce very few false positives. However, some routers were unresponsive to alias resolution probes (e.g., Google) or sent constant or random IP-ID values, so false negatives are possible. Alias resolution helped us improve the accuracy of our IP-to-ASN mappings, but more importantly it provided additional constraints for mapping interfaces to facilities.

\subsection{Constrained Facility Search}

At the end of the previous step we obtained three representations of the routing paths between the vantage points and our targets: the IP-level paths as well as the corresponding router-level and AS-level abstractions. We combined this topology data with the map-

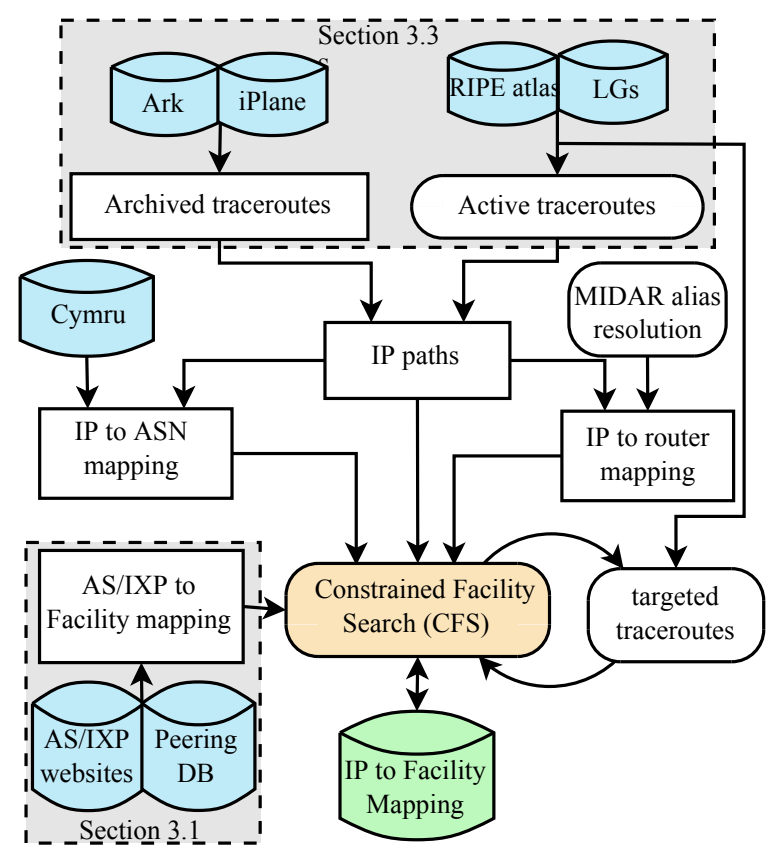

Figure 4: Overview of our interconnection-to-facility mapping process. Using a large set of traceroutes toward the target ASes, we first resolve IP addresses to routers [40], and map IP addresses to ASes [21]. We feed this data, along with a constructed list of interconnection facilities for each AS and IXP, to our Constrained Facility Search (CFS) algorithm. The CFS algorithm iteratively constrains the possible interconnection facilities for inferred peerings, using targeted traceroutes as necessary to narrow the possible facilities for a router until it converges.

ping of ASes and IXPs to facilities that we constructed in section 3.1.1. We use the combined data to infer the location of the targeted peering interconnections as follows. We progressively constrain the possible facilities where an IP interface is located, through a process of triangulation similar to those used in RTT-based geolocation [33, 37], but instead of delay data we rely on facility information data. Figure 4 provides an overview of the Constrained Facility Search (CFS) algorithm. We use the notation in table 2 to describe this algorithm.

Step 1: Identifying public and private peering interconnections. Consider the case where we want to infer the facilities where peers of AS B interconnect. We start by searching recently collected traceroute data for peering interconnections involving B. If we find such an interconnection, we then identify the peering interface of the neighbor AS involved in the interconnection and whether the peering is public or private, using the following technique.

If we observed a sequence of IP hops $\left(I P_{A}, I P_{e}, I P_{B}\right)$, in a traceroute path, we check if interface $I P_{e}$ belongs to the address space of an IXP that is used for public peering, assembled as explained in section 3.1.2. If $I P_{e}$ 


\begin{tabular}{|l|l|}
\hline Notation & Meaning \\
\hline$I P_{x}^{i}$ & $\begin{array}{l}\text { The } i \text { th IP interface that is } \\
\text { mapped to ASN } x .\end{array}$ \\
\hline$\left(I P_{x}, I P_{y}, I P_{z}\right)$ & $\begin{array}{l}\text { Sequence of IP hops in a } \\
\text { traceroute path. }\end{array}$ \\
\hline$\left\{F_{A}\right\}$ & $\begin{array}{l}\text { The set of interconnection facilities } \\
\text { where ASN A is present. }\end{array}$ \\
\hline$I P_{x} \rightarrow\left\{f_{1}, f_{2}\right\}$ & $\begin{array}{l}\text { The IP interface } I P_{x} \text { is mapped to } \\
\text { either facility } f_{1} \text { or facility } f_{2}\end{array}$ \\
\hline
\end{tabular}

Table 2: Notation used in Constrained Facility Search.

belongs to IXP address space, we infer that the peering link $(A, B)$ is public and is established over the IXP that owns $I P_{e}$ [10]. If $I P_{e}$ is an unresolved (no mapping to AS) or an unresponsive interface, we discard the path. If we observed a sequence of IP hops $\left(I P_{A}, I P_{B}\right)$, then we infer that the peering interconnection $(A, B)$ is private, since there is no intermediate network between AS A and AS B. This peering interconnection can be either cross-connect, tethering, or remote.

Step 2: Initial facility search. After we determine the public and private peering interconnections, we calculate the possible locations where each interconnection could be established. This step allows us to create an initial set of candidate locations for each peering interface, and helps us to distinguish cross-connects from remote peering and tethering. For a public peering $\left(I P_{A}, I P_{I X P}, I P_{B}\right)$ we compare the set of facilities $\left\{F_{A}\right\}$ where AS A has presence with the IXP's set of interconnection facilities $\left\{F_{E}\right\}$. Note that we can compare the facilities between AS A and IXP, but not the facilities between IXP and AS B, because typically the traceroute response returns from the ingress interface and therefore the interface of AS B at the IXP is not visible in the traceroute paths. From this comparison we have three possible outcomes regarding the resolution of an IP interface to facility:

1. Resolved interface: If AS A has only one common facility with the IXP, we infer that AS A is not remotely interconnected to the IXP, and that its interface $I P_{A}$ is located in the common facility.

2. Unresolved local interface: If AS A has multiple common facilities with the IXP, then we infer that AS A is not remotely interconnected to the IXP, and that its interface $I P_{A}$ is located in one of the common facilities, i. e., $I P_{A} \rightarrow\left\{\left\{F_{A}\right\} \cap\left\{F_{E}\right\}\right\}$.

3. If AS A has no common facility with the IXP, then we have two possibilities: (a) Unresolved remote interface: AS A is remotely connected to the IXP through a remote peering reseller. For these interfaces the set of possible facilities includes all facilities where AS A is present, i. e., $I P_{A} \rightarrow\left\{F_{A}\right\}$. (b) Missing data: We have incomplete facility data about AS A or about the IXP that prevents inference of the location of the interconnection.
We used the methodology developed in [14] to infer remote peering. We used multiple measurements taken at different times of the day to avoid temporarily elevated RTT values due to congestion.

We performed a similar process for private peering interconnections $\left(I P_{A}, I P_{B}\right)$, but this time we calculated the common facilities between private peers AS A and AS B. Again, there are three possible outcomes: (1) a single common facility in which we infer $I P_{A}$ to be located, (2) multiple common facilities where $I P_{A}$ may be located, or (3) no common facilities, meaning that the interconnection is remote private peering or tethering, or we have missing data.

Step 3: Constraining facilities through alias resolution. Identifying the facility of an interface means that all aliases of that interface should be located in the same facility. This observation allows us to further constrain the set of candidate facilities for unresolved interfaces in the previous step (unresolved local or remote). Even if none of a router's aliases were resolved in the previous step to a single router, it is possible that by cross-checking the candidate facilities of the aliases we will converge to a single facility for all of the aliases. For example, consider two unresolved interfaces $I P_{A}^{1}$ and $I P_{A}^{2}$, with $I P_{A}^{1} \rightarrow\left\{f_{1}, f_{2}\right\}$ and $I P_{A}^{2} \rightarrow\left\{f_{2}, f_{3}\right\}$. Since $I P_{A}^{1}$ and $I P_{A}^{2}$ are aliases, it means that they can only be located at the common facilities, hence we infer that both aliases are located in facility $f_{2}$.

Step 4: Narrowing set of facilities through follow-up targeted traceroutes. For the remaining unresolved interfaces, we need to perform additional traceroute measurements in order to further constrain the set of possible facilities [12]. For an unresolved interface, our goal is to find different peering connections involving the interface so that we obtain sufficient constraints to infer a facility. We carefully selected targets of these follow-up traceroutes to increase the likelihood of finding additional constraints. Selection of targets depends on the type of unresolved interface, and the already inferred possible facilities, both of which we determined in Step 2.

For an unresolved local peering interface $I P_{A}^{x}$ we need to target other ASes whose facilities overlap with at least one candidate facility of $I P_{A}^{x}$. However, it is important that the candidate facilities of $I P_{A}^{x}$ are a superset of the target's facilities. Otherwise, comparison of the common facilities between $A$ and the target will not further constrain the already inferred facilities for $I P_{A}^{x}$. We select as follow-up traceroute targets IP addresses in ASes with $\left\{F_{\text {target }}\right\} \subset\left\{F_{A}\right\}$, and we launch follow-up traceroutes starting from the target with the smallest facility overlap. The resulting traceroute will contribute constraints only if it does not cross the same IXP against which we compared the facilities of $A$ in Step 1. Therefore, we need to find paths that cross private peering interconnections or previously unseen IXPs, which means that we prioritize targets that are not colocated in the already queried IXP. 


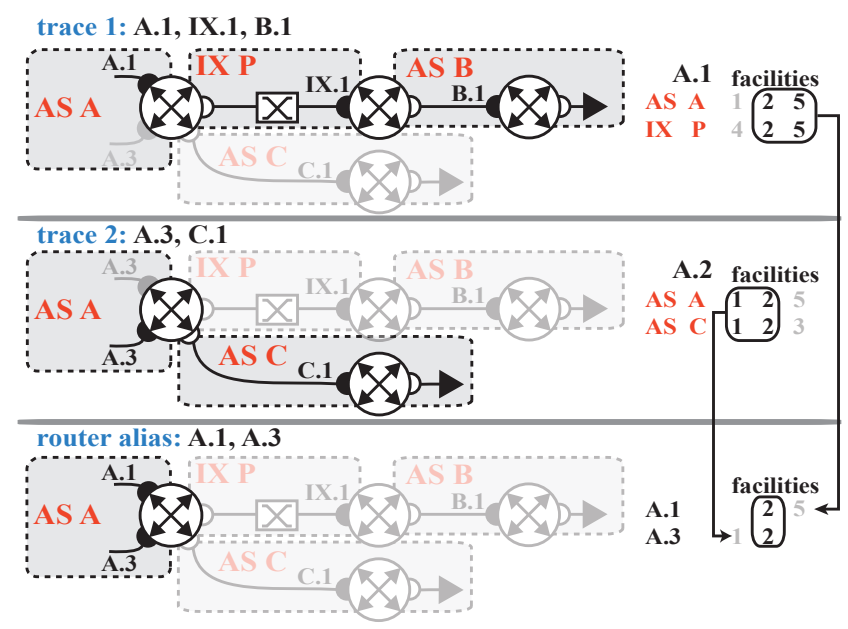

Figure 5: Toy example of how we use Constrained Facility Search (CFS) method to infer the facility of a router by probing the interconnection between peers with known lists of colocation facilities (described in detail at end of Section 4.2).

An unresolved remote interface $I P_{A}^{y}$ occurs when Step 1 does not provide any constraints, meaning that the possible facilities for $I P_{A}^{y}$ are the entire set of facilities where $A$ is present. In this case, we intend the targeted traceroutes to find local peering interconnections (public or private) that involve the router of $I P_{A}^{y}$. Again, we begin the measurements from the ASes with the smallest possible non-empty overlap of facilities.

After we launch the additional targeted traceroute measurements, we repeat Steps 2 to 4 until each interface converges to a single facility, or until a timeout set for searching expires. To illustrate the execution of our algorithm consider the example in Figure 5. Using trace 1 and by comparing the common facilities between AS $A$ and IXP we find that the interface $A .1$ is located in Facility 2 or Facility 5. Similarly, using trace 2 and by comparing the common facilities between AS $A$ and AS $B$ we find that the interface $A .3$ is located in Facility 1 or Facility 2. To further narrow the potential facilities where the interfaces $A .1$ and $A .3$ are located, we de-alias the collected interfaces and map the resulting router to the intersection of the candidate facilities for each interface. At the end of this process we infer that the interfaces $A .1$ and $A .3$ are located in facility 2 .

\subsection{Facility search in the reverse direction}

So far we have located the peering interconnections from the side of the peer AS that appears first in the outgoing direction of the traceroute probes. In some cases, due to the engineering approach to interconnection (i. e., private cross-connect), it is expected that the second peer (the other side of the interconnection) is in the same facility or building. Furthermore, in many cases of public peering interconnections over IXPs, the unresolved peer is connected to only a single IXP facil-

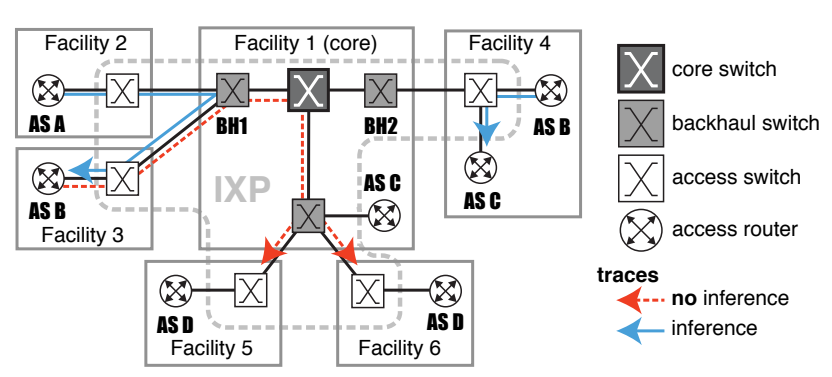

Figure 6: Toy example to illustrate the execution of the Switch Proximity Heuristic (Section 4.4) to infer the interconnection facility of the peer at the far end of an IXP peering link when the peer is connected in more than one IXP facility.

ity. However, in remote peering, tethering and public peering at IXPs where the second peer is connected at multiple facilities, the two peers can be located at different facilities. For example, in Figure 5 the CFS algorithm will infer the facility of A.1's router but not the facility of IX.1's router. This outcome arises because traceroute replies typically return from the ingress, black, interface of a router and therefore do not reveal the router's exgress, white, interfaces.

To improve our visibility, Steps 1-4 can also be repeated in the reverse paths, if we have a monitor at the side of the second peer. For many cases, but not all, this reverse search is possible, because we use a diverse set of vantage points. But obtaining reverse paths using the record route option and correlating traceroutes [38] is not a general solution to the problem and cannot be applied to interconnections with several popular content providers.

\subsection{Proximity Heuristic}

As a fallback method to pinpoint the facility of the far end interface, we use knowledge of common IXP practices with respect to the location and hierarchy of switches. We confirmed with operators via private communication that networks connected to the same switch, or connected to switches attached with the same backhaul switch, exchange traffic locally and not via the core switch. For example, consider the toy IXP setup of Figure 6, over which AS $A$ establishes a public peering with AS $B$. AS $A$ will send its traffic to the router of AS $B$ in Facility 3 instead of Facility 4, because Facilities 2 and 3 are connected on the same backhaul switch (BH1), while Facilities 2 and 4 would need to exchange traffic over the Core switch. Consequently, we develop the switch proximity heuristic. For a public peering link $\left(I P_{A}, I P_{I X P, B}, I P_{B}\right)$ for which we have already inferred the facility of $I P_{A}$, and for which $I P_{B}$ has more than one candidate IXP facilities, we require that $I P_{B}$ is located in the facility that is proximate to $I P_{A}$.

Given that a detailed switch topology of an IXP is not always available, we infer the proximity of the IXP 
facilities through probabilistic ranking based on the IPto-facility mapping that we performed in the previous steps. More specifically, for each IXP facility that appears at the near end of a public peering link (e.g. facility of $A_{1}$ in Figure 5), we count how often it traverses a certain IXP facility at the far end (facility of $B_{1}$ in Figure 5) whenever the far end has more than one candidate facility, and we rank the proximity of IXP facilities using this metric. Based on the inferred proximities we then try to determine the facilities of public peering links for which we do not have traceroute paths from the reverse direction. If we have pinpointed the facility of the near-end peering router, we require that the farend router will be located in the most proximate facility to the near-end router.

We validated inferences from the switch proximity heuristic against ground-truth data from AMS-IX [1], which provides both the interfaces of the connected members and the corresponding facilities of those interfaces. We executed an additional traceroute campaign from 50 AMS-IX members who are each connected to a single facility of AMS-IX, targeting a different set of 50 AMSIX members who are each connected to two facilities. We found that in $77 \%$ of the cases the switch proximity heuristic finds the exact facility for each IXP interface. When it fails, the actual facility is in close proximity to the inferred one (e.g., both facilities are in the same building block), which is because (per the AMS-IX web site) the access switches are connected with the same backhaul switch. Moreover, the heuristic cannot make inferences when the potential facilities are connected at the same backhaul or core switch. For example, for traffic between AS $B$ and AS $D$ in Figure 6, we cannot infer the facility of AS $D$ because both facilities of AS $D$ have the same proximity to the facilities of AS $B$.

\section{RESULTS}

To evaluate the feasibility of our methodology, we first launched an $\mathrm{IPv} 4$ traceroute campaign from different measurement platforms targeting a number of important groups of interconnections, and tried to infer their locations. We considered a number of popular content providers whose aggregated traffic is responsible for over half the traffic volume in North America and Europe by some accounts [30, 51]: Google (AS15169), Yahoo! (AS10310), Akamai (AS20940), Limelight (AS22822) and Cloudflare (AS13335). For these networks we maintain a list of their IP prefixes via their BGP announcements (in some cases a content provider uses more than one ASN) as well as white lists that they make available to avoid blocking by firewalls [17] or provided by previous studies [13]. We also collected a list of URLs they deliver [8] that we can use for our traceroute campaigns. If the traceroute targets are IPs, we made sure that we used active IPs, relying on the list from ZMap [26] as well as a ping immediately before finalizing the list. Before using any of the URLs, we checked that the URL was available and delivered by the expected content de- livery network.

Second, we considered four large transit providers (the main ASN that they use to peer is in parenthesis) with global footprints: NTT (AS2914), Cogent (AS174), and Deutsche Telekom (AS3320), Level3 (AS3356) and Telia (AS1299). We collected the list of all their peers and augmented this list with the names of PoPs available on the four networks' web sites. Again, we selected one active IP per prefix for each peers.

In total we were able to map 9,704 router interfaces to a single interconnection facility, after completing 100 iterations of $\mathrm{CFS}^{1}$. Figure 7 shows the number of iterations of the CFS algorithm needed for the collected interfaces to converge to a single facility.

At the end of the first iteration we obtain the interfaceto-facility resolutions that correspond to peering ASes with only a single common facility. Most remote peering interconnections were resolved in step 2, as an outcome of CFS changing the traceroute target to facilities that are local to the remote peer. About $40 \%$ of the interfaces are resolved after the first 10 iterations, while we observe diminishing returns after 40 iterations of the algorithm. We defined the timeout at 100 rounds, after which we managed to pinpoint $70.65 \%$ of the peering interfaces. For about $9 \%$ of the unresolved interfaces we were able to constrain the location of the interface to a single city. We also study the convergence rate when we use only a single platform for targeted measurements, either RIPE Atlas or Looking Glasses. As shown in Figure 7, RIPE Atlas infers two times more interfaces per iteration when compared with Looking Glasses. However, $46 \%$ of the interfaces inferred by the Looking Glasses, many of which are located at the backbone of large transit provider, are not visible to the Atlas probes.

To put our convergence rate into context, we attempted to geolocate the collected interfaces using DRoP [34], a DNS-based geolocation technique that extracts geographically meaningful information from DNS hostnames, such as airport codes, city names, and CLLI codes. From the 13,889 peering interfaces in our traceroute data, $29 \%$ have no associated DNS record, while $55 \%$ of the remaining 9,861 interfaces do not encode any geolocation information in their hostname. Ultimately, we were able to geolocate only $32 \%$ of the peering interfaces using DNS geolocation. This percentage is smaller than the first 5 iterations of the CFS algorithm, and it provides more coarse-grained geolocation.

For $33 \%$ of the interfaces that were not resolved to a facility, we did not have any facility information for the AS that owns the interface address. To understand better the effect of missing facility data on the fraction of resolved interfaces, we executed CFS while iteratively removed 1,400 facilities from our dataset in random order and we repeated this experiment 20 times. As shown in Figure 8, removing 850 facilities ( $50 \%$ of the total fa-

\footnotetext{
${ }^{1}$ Each iteration of the CFS algorithm repeats steps 2-4 as explained in section 4.2 .
} 

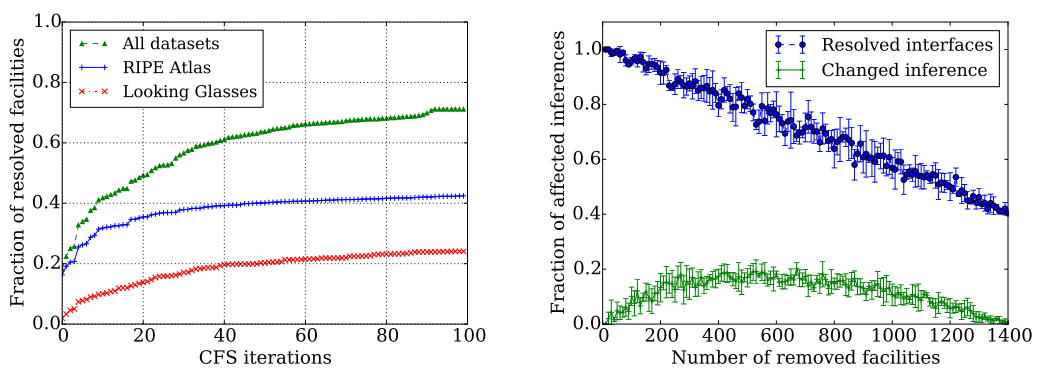

Figure 7: Fraction of resolved in- Figure 8: Average fraction of unterfaces versus number of CFS it- resolved interfaces, and interfaces erations when we use all, RIPE At- with erroneous facility inference by las, or LG traceroute platforms.

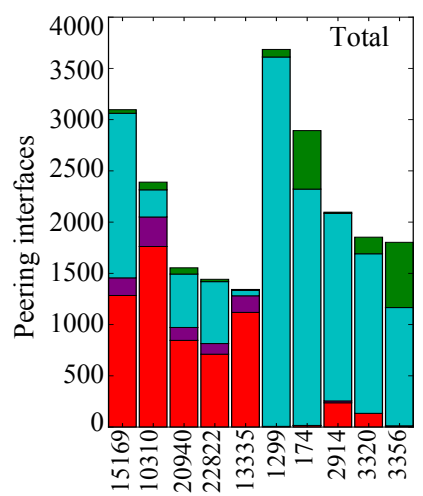

iteratively removing 1400 facilities.

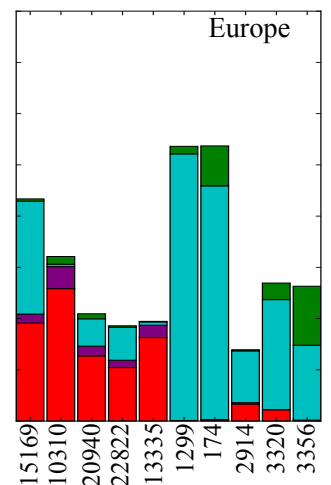

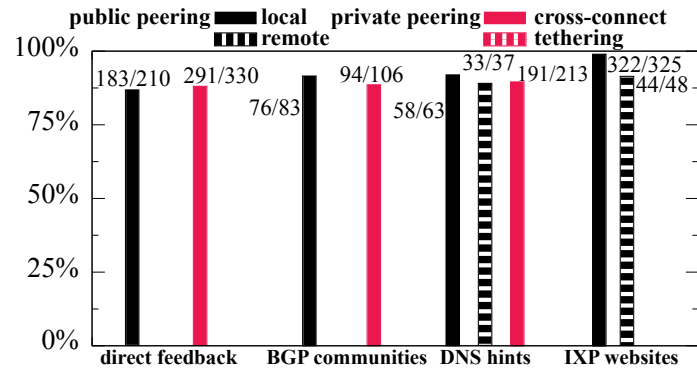

Figure 9: Fraction of ground truth locations that match inferred locations, classified by source of ground truth and type of link inferred. CFS achieves $90 \%$ accuracy overall.
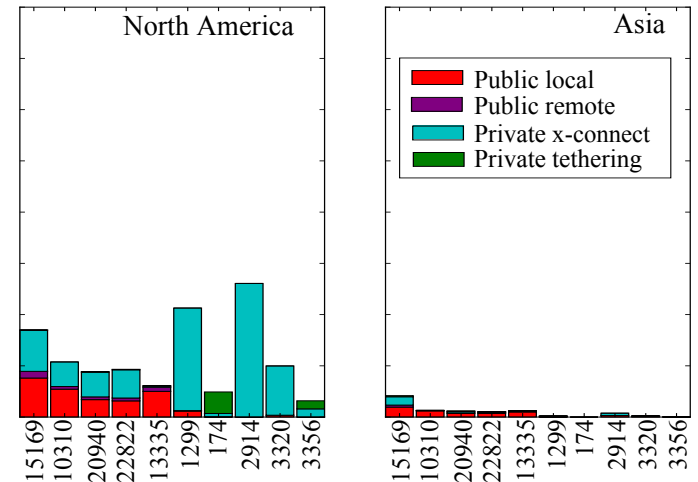

Figure 10: Number of peering interfaces inferred and distribution by peering type for a number of networks in our study around the globe and per region.

cilities in our dataset) causes on average $30 \%$ of the previously resolved interfaces to become unresolved, while when we remove $1,400(80 \%)$ facilities $60 \%$ of the resolved interfaces become unresolved. Although, missing facility data can significantly reduce the completeness of the IP-to-facility resolution, even when removing a large majority of the facilities in our dataset the completeness of CFS IP-to-facility mapping is comparable to that of IP-to-city DNS-based geolocation.

Incomplete data can also affect the correctness of our inferences. Missing facility information can cause the CFS algorithm to incorrectly converge to a single facility, by cross-referencing incomplete datasets. For instance, if in Figure $5 \mathrm{AS} \mathrm{C}$ is also present in Facility 5 , then it is possible that the interfaces A.1 and A.2 are located in Facility 5 but the algorithm converges to Facility 2 because it does not have this information. As shown Figure 8, removing 500 (30\%) of the facilities in our dataset can cause $20 \%$ of the interfaces to converge to a different facility (changed inference). Interestingly, the number of removed facilities does not have a monotonic relationship with the number of changed inferences. Removing over $30 \%$ of the facilities violates one of the requirements for the CFS to resolve an interface to a facility, i.e., the existence of sufficient constraints to narrow the search.
An interesting outcome of our measurements is that $39 \%$ of the observed routers were used to implement both public and private peering. Although we cannot generalize this finding since our traceroute campaign did not aim an exhaustive discovery of peering interconnections, the large fraction of the observed multi-role routers implies that private and public peering interconnections often rely on the same routing equipment and can have common points of congestion or failure. Therefore, public and private peering interconnections are more interdependent than previously believed. We also find that $11.9 \%$ of the observed routers used to implement public peering, are used to establish links over two or three IXPs. As explained in section 3.1.1, an interconnection facility can be used from more than one IXPs as colocation points, therefore a router located in a cross-IXP facility can be used to establish links with peers across all the IXPs colocated at the same facility. Importantly, this heterogeneity helps our algorithm to resolve a higher number of interfaces to facilities, since we can use a mix of IXPs and private peering facilities to constrain the possible facilities for the multi-role router interfaces.

In Figure 10 we present the total number of peering interfaces per target AS and how they are distributed in three major regions (Europe, North America, and 
Asia). We are aware that there are different business practices that may affect the inference and localization of the interfaces in our study. For example, information regarding facility locations and business relationships is less transparent in the US than in Europe. We also attribute differences due to the distribution of the vantage points we utilize in our study. For example, RIPE Atlas probes have a significantly larger footprint in Europe than in Asia, thus, it is expected that we can infer more interfaces in Europe. Regarding the qualitative differences among different types of networks, it is pronounced that CDNs establish a major fraction of their peering interconnections over public peering fabric, in contrast to Tier-1 networks. However, even among Tier1 networks we observe significant variance in peering strategies.

\section{VALIDATION}

Due to its low-level nature, ground-truth data on interconnection to facility mapping is scarce. We tried to validate our inferences as extensively as possible by combining four different sources of information:

Direct feedback: We obtained direct validation from two of the CDN operators we used as targets for our measurements. The operators offered validation only for their own interfaces but not the facilities of their peers due to lack of data. Overall, we validated $88 \%$ $(474 / 540)$ of our inferences as correct at the facility level, and $95 \%$ as correct at the city level. Missing facilities from our colocation dataset were responsible for $70 \%$ of the wrong inferences.

BGP communities: AS operators often use the BGP communities attribute to tag the entry point of a route in their network. The entry point to the AS network is at the near end of the peering interconnection, for which we made facility inferences, meaning that the communities attribute can be used as a source of validation. We compiled a dictionary of 109 community values used to annotate ingress points, defined by 4 large transit providers. For our validation we use only data from looking glasses that provide BGP and traceroute vantage points from the same routers, to avoid path disparities due to potential path diversity between different PoPs of an AS. We queried the BGP records of addresses that we used as destinations in our traceroute measurements, and we collected the communities values attached to the BGP routes. Of the inferences validated, we correctly pinpointed $76 / 83(92 \%)$ of public peering interfaces and 94/106 (89\%) of cross-connect interfaces.

DNS records: Some operators encode the facility of their routers in the hostnames of the router interfaces. For example the hostname $\mathrm{x} \cdot \mathrm{y} \cdot \mathrm{rtr}$. thn. Ion.z denotes that a router is located in the facility Telehouse-North in London. We compiled a list of naming conventions that denote interconnection facilities from 7 operators in the UK and Germany, and we confirmed with them that the DNS records were current. Of the interfaces vali- dated, we correctly pinpointed 91/100 (91\%) of public peering interfaces and 191/213 (89\%) of cross-connect interfaces.

IXP websites: A few IXPs list in their websites the exact facilities and the IP interfaces where their members are connected. We collected data from 5 large European IXPs (AMS-IX, NL-IX, LINX, France-IX and STH-IX) and compared them against our inferences. AMS-IX and France-IX also distinguished between local and remote peers. Although they provided the location of their reseller, we used the data to verify that our remote-peering inferences were correct. Of the interfaces validated, we correctly pinpointed 322/325 (99.1\%) of public peering interfaces correctly inferred 44/48 (91.7\%) of remote peers. The higher accuracy rate for this validation subset is explained by the fact that we collected through the IXP websites complete facilities lists for the IXPs and their members.

As shown in figure 9, the CFS algorithm correctly pinpointed correctly over $90 \%$ of the interfaces. Importantly, when our inferences disagreed with the validation data the actual facility was located in the same city as the inferred one (e. g., Telecity Amsterdam 1 instead of Telecity Amsterdam 2).

\section{RELATED WORK}

Several research efforts have mapped peering interconnections to specific regions using a variety of data sources. Augustin et al. [10] used traceroute measurements to infer peering interconnections established at IXPs. Dasu [56] used BitTorrent clients as vantage points to accomplish a similar mapping. Follow-up work [32] used public BGP information from IXP route servers to infer a rich set of additional peerings. The above studies map interconnections to a specific city where an IXP operates. Castro et al. [14] provided a delaybased method to infer remote peerings of IXP members, i.e., to infer which of the IXP members utilize remote routers to exchange traffic. Giotsas et al. [31] used BGP communities and information about measurement vantage points to map interconnections at the city level. Calder et al. [13] provided novel techniques based on delay measurements to locate peerings and points of presence of a large content provider at the city level. A recent effort by Motamedi et al. [48] proposed a methodology that combines public BGP data, and geographically-constrained measurements in data plane (targeted traceroute campaigns) and control plane (BGP records from selective BGP-enabled looking glass servers) for mapping the cross connects in commercial colocation facilities. They demonstrated that it is feasible to accurately localize the cross connects of all the tenants for two target facilities they considered in their study.

Two other methods are widely used to infer the location of an interconnection: reverse DNS lookup and IP geolocation. A major problem with the first method is that DNS entries are not available for many IP addresses involved in interconnections, including Google's. 
Furthermore, when a DNS entry exists, it is challenging to engineer the set of regular expression patterns to infer the manual rules used by different providers. Furthermore, many providers do not regularly maintain or update DNS entries, thus, DNS entries may be misleading $[62,29]$. On the other hand, IP geolocation is known for its inaccuracy, and studies have shown that it can be reliable only at the country or state level [52, $35,33]$. Due to the inaccuracy of many geolocation services, in some cases, e.g., Google, all IP addresses of prefixes used for interconnection will map to California, even when those IP addresses are clearly more geographically distributed.

Another recent study [25] provides techniques to infer all intra-ISP links at the point of presence (PoP) level and discovered more nodes and links than was previously possible with network layer measurements [58]. Given a physical location, this probing technique maps the collected IP interfaces to city-level PoPs using the dataset in [24] to extract geolocation hints from DNS hostname. While the focus of this study is not inferring the location of peering interconnections, it provided useful insights related to the design and execution of traceroute campaigns. Research projects have also assembled physical Internet maps of ISPs at the PoP level [42] and of long-haul fiber-optic network in the U.S. [23].

\section{CONCLUSION}

The increasing complexity of interconnection hinders our ability to answer questions regarding their physical location and engineering approach. But this capability - to identify the exact facility of a given interconnection relationship, as well as its method - can enhance if not enable many network operations and research activities, including network troubleshooting, situational awareness and response to attacks, and many Internet cartography challenges.

In this paper we presented a measurement-driven methodology, called constrained facility search, to infer the physical facility where a target interconnection occurs from among all possible candidates, as well as the type of interconnection used. Our process narrowed the number of candidate facilities for a given interconnection to those consistent with other known interconnections involving the same routers. Eventually the multiple sources of constraints led to a small enough set of possible peering locations that in many cases, it became feasible to identify a single location that satisfied all known constraints. We believe this is the first time that these types of constraints have been used to infer where an interconnection physically occurs. The accuracy of our method (>90\%) outperforms heuristics based on naming schemes and IP geolocation.

We emphasize that discovery of all interconnections in the Internet is a far-reaching goal; our method can infer the location and type of given interconnections. Nevertheless, by utilizing results for individual interconnections and others inferred in the process, it is pos- sible to incrementally construct a more detailed map of interconnections. We make our data available at http:// www.caida.org/publications/paper/2015/constrained_facility_ search/supplemental

\section{ACKNOWLEDGMENTS}

This work was supported in part by NSF CNS-1414177. Georgios Smaragdakis was supported by the EU Marie Curie IOF "CDN-H" (PEOPLE-628441).

\section{REFERENCES}

https://ams-ix.net/connected_parties.

[2] DE-CIX quick facts. http://www.de-cix.net/about/quick-facts.

[3] Equinix Investor Relations: Annual and Quarter Results. http://investor.equinix.com/.

[4] Google Peering Policy (accessed 2013-4). https: //peering.google.com/about/peering_policy.html.

[5] JPNAP Tokyo I Service. http: //www.jpnap.net/english/jpnap-tokyo-i/index.html.

[6] The Internet Under Crisis Conditions: Learning from September 11. The National Academy Press, 2003.

[7] B. Ager, N. Chatzis, A. Feldmann, N. Sarrar, S. Uhlig, and W. Willinger. Anatomy of a Large European IXP. In ACM SIGCOMM, 2012.

[8] B. Ager, W. Mühlbauer, G. Smaragdakis, and S. Uhlig. Web Content Cartography. In $A C M I M C$, 2011.

[9] B. Augustin, X. Cuvellier, B. Orgogozo, F. Viger, T. Friedman, M. Latapy, C. Magnien, and R. Teixeira. Avoiding traceroute anomalies with Paris traceroute. In $A C M I M C, 2006$.

[10] B. Augustin, B. Krishnamurthy, and W. Willinger. IXPs: Mapped? In $A C M I M C, 2009$.

[11] Broadband Internet Technical Advisory Group Report (BITAG). Interconnection and Traffic Exchange on the Internet, 2014.

[12] R. Bush, O. Maennel, M. Roughan, and S. Uhlig. Internet Optometry: Assessing the Broken Glasses in Internet Reachability. In ACM IMC, 2009.

[13] M. Calder, X. Fan, Z. Hu, E. Katz-Bassett, J. Heidemann, and R. Govindan. Mapping the Expansion of Google's Serving Infrastructure. In $A C M$ IMC, 2013.

[14] I. Castro, J. C. Cardona, S. Gorinsky, and P. Francois. Remote Peering: More Peering without Internet Flattening. In CoNEXT, 2014.

[15] I. Castro and S. Gorinsky. T4P: Hybrid Interconnection for Cost Reduction. In NetEcon, 2012.

[16] H. Chang, S. Jamin, and W. Willinger. Inferring AS-level Internet topology from router-level path traces. In ITCom, 2001.

[17] N. Chatzis, G. Smaragdakis, J. Boettger, T. Krenc, and A. Feldmann. On the benefits of using a large IXP as an Internet vantage point. In ACM IMC, 2013.

[18] N. Chatzis, G. Smaragdakis, A. Feldmann, and W. Willinger. There is More to IXPs than Meets the Eye. ACM CCR, 43(5), 2013.

[19] N. Chatzis, G. Smaragdakis, A. Feldmann, and W. Willinger. Quo vadis Open-IX? Trying to boost public peering in the US. ACM CCR, 45(1), 2015.

[20] K. Cho, C. Pelsser, R. Bush, and Y. Won. The Japan Earthquake: the impact on traffic and routing 
observed by a local ISP. In ACM CoNEXT SWID workshop, 2011.

[21] Cymru Team. IP to ASN mapping.

http://www.team-cymru.org/IP-ASN-mapping.html.

[22] J. Czyz, M. Kallitsis, M. Gharaibeh, C. Papadopoulos, M. Bailey, and M. Karir. Taming the 800 Pound Gorilla: The Rise and Decline of NTP DDoS Attacks. In $A C M I M C, 2014$.

[23] R. Durairajan, P. Barford, J. Sommers, and W. Willinger. InterTubes: A Study of the US Long-haul Fiber-optic Infrastructure. In $A C M$ SIGCOMM, 2015.

[24] R. Durairajan, S. Ghosh, X. Tang, P. Barford, and B. Eriksson. Internet atlas: A Geographic Database of the Internet. In Proc. of HotPlanet, 2013.

[25] R. Durairajan, J. Sommers, and P. Barford. Layer 1-Informed Internet Topology Measurement. In $A C M$ IMC, 2014.

[26] Z. Durumeric, E. Wustrow, and J. A. Halderman. ZMap: Fast Internet-Wide Scanning and its Security Applications. In USENIX Security Symposium, 2013.

[27] Equinix. Global Data Centers and Colocation Services. http://www.equinix.com/locations/.

[28] P. Faratin, D. Clark, S. Bauer, W. Lehr, P. Gilmore, and A. Berger. The Growing Complexity of Internet Interconnection. Communications and Strategies, 2008.

[29] M. J. Freedman, M. Vutukuru, N. Feamster, and H. Balakrishnan. Geographic Locality of IP Prefixes. In $A C M I M C, 2005$.

[30] A. Gerber and R. Doverspike. Traffic Types and Growth in Backbone Networks. In OFC/NFOEC, 2011.

[31] V. Giotsas, M. Luckie, B. Huffaker, and kc claffy. Inferring Complex AS Relationships. In ACM IMC, 2014.

[32] V. Giotsas, S. Zhou, M. Luckie, and kc claffy. Inferring Multilateral Peering. In CoNEXT, 2013.

[33] B. Gueye, A. Ziviani, M. Crovella, and S. Fdida. Constraint-Based Geolocation of Internet Hosts. IEEE/ACM Trans. Networking, 14(6), 2006.

[34] B. Huffaker, M. Fomenkov, et al. Drop: Dns-based router positioning. ACM SIGCOMM Computer Communication Review, 44(3):5-13, 2014.

[35] B. Huffaker, M. Fomenkov, and k. claffy. Geocompare: A Comparison of Public and Commercial Geolocation Databases. Technical report, CAIDA, May 2011.

[36] Interxion. Locations. http://www.interxion.com/locations/.

[37] E. Katz-Bassett, J. P. John, A. Krishnamurthy, D. Wetherall, T. Anderson, and Y. Chawathe. Towards IP geolocation using delay and topology measurements. In ACM IMC, 2006.

[38] E. Katz-Bassett, H. Madhyastha, V. Adhikari, C. Scott, J. Sherry, P. van Wesep, A. Krishnamurthy, and T. Anderson. Reverse Traceroute. In NSDI, 2010.

[39] T. Kernen. traceroute.org. http://www.traceroute.org.

[40] K. Keys, Y. Hyun, M. Luckie, and k claffy. Internet-Scale IPv4 Alias Resolution with MIDAR. IEEE/ACM Trans. Networking, 21(2), 2013.

[41] A. Khan, T. Kwon, H. C. Kim, and Y. Choi. AS-level Topology Collection through Looking Glass Servers. In ACM IMC, 2013.

[42] S. Knight, H. X. Nguyen, N. Falkner, R. Bowden, and M. Roughan. The Internet Topology Zoo. IEEE J. on Sel. Areas in Comm., 29(9), 2011.
[43] A. Kumar, V. Paxson, and N. Weaver. Exploiting Underlying Structure for Detailed Reconstruction of an Internet Scale Event. In ACM IMC, 2005.

[44] C. Labovitz, S. Lekel-Johnson, D. McPherson, J. Oberheide, and F. Jahanian. Internet Inter-Domain Traffic. In ACM SIGCOMM, 2010.

[45] A. Lodhi, N. Larson, A. Dhamdhere, C. Dovrolis, and K. Claffy. Using PeeringDB to Understand the Peering Ecosystem. ACM CCR, 44(2), 2014.

[46] M. Luckie, A. Dhamdhere, D. Clark, B. Huffaker, and kc claffy. Challenges in Inferring Internet Interdomain Congestion. In ACM IMC, 2014.

[47] H. V. Madhyastha, T. Isdal, M. Piatek, C. Dixon, T. Anderson, A. Kirshnamurthy, and A. Venkataramani. iPlane: An information plane for distributed systems. In ACM OSDI, 2006.

[48] R. Motamedi, B. Chandrasekaran, B. Maggs, R. Rejaie, and W. Willinger. On the Geography of X-Connects. U. Oregon, CIS-TR-2014-1, May 2015.

[49] W. Muhlbauer, A. F. O. Maennel, M. Roughan, and S. Uhlig. Building an AS-Topology Model that Captures Route Diversity. In ACM SIGCOMM, 2006.

[50] W. B. Norton. The Art of Peering: The Peering Playbook, 2010.

[51] I. Poese, B. Frank, G. Smaragdakis, S. Uhlig, A. Feldmann, and B. Maggs. Enabling Content-aware Traffic Engineering. ACM CCR, 42(5), 2012.

[52] I. Poese, S. Uhlig, M. A. Kaafar, B. Donnet, and B. Gueye. IP Geolocation Databases: Unreliable? ACM CCR, 41(2), 2011.

[53] L. Quan, J. Heidemann, and Y. Pradkin. Trinocular: Understanding Internet Reliability Through Adaptive Probing. In ACM SIGCOMM, 2013.

[54] P. Richter, G. Smaragdakis, A. Feldmann, N. Chatzis, J. Boettger, and W. Willinger. Peering at Peerings: On the Role of IXP Route Servers. In $A C M I M C$, 2014.

[55] M. Roughan, W. Willinger, O. Maennel, D. Pertouli, and R. Bush. 10 Lessons from 10 Years of Measuring and Modeling the Internet's Autonomous Systems. IEEE J. on Sel. Areas in Comm., 29(9), 2011.

[56] M. A. Sánchez, J. S. Otto, Z. S. Bischof, D. R. Choffnes, F. E. Bustamante, B. Krishnamurthy, and W. Willinger. Dasu: Pushing Experiments to the Internet's Edge. In NSDI, 2013.

[57] J. H. Sowell. Framing the Value of Internet Exchange Participation. In Proc. of TPRC, 2013.

[58] N. Spring, R. Mahajan, and D. Wetherall. Measuring ISP topologies with Rocketfuel. In ACM SIGCOMM, 2002.

[59] Telehouse. Colocation Services. http://www.telehouse.com/facilities/colocation/.

[60] R. van Rijswijk-Deij, A. Sperotto, and A. Pras. DNSSEC and its Potential for DDoS Attacks - A Comprehensive Measurement Study. In $A C M I M C$, 2014.

[61] W. Willinger and M. Roughan. Internet Topology Research Redux. Recent Advances in Networking, 1(1), 2013.

[62] M. Zhang, Y. Ruan, V. Pai, and J. Rexford. How DNS Misnaming Distorts Internet Topology Mapping. In USENIX ATC, 2006.

[63] Y. Zhang, R. Oliveira, H. Zhang, and L. Zhang. Quantifying the pitfalls of traceroute in AS connectivity inference. In $P A M, 2010$. 